\title{
Effect of rifampicin and clarithromycin on the CYP3A activity in patients with Mycobacterium avium complex
}

\author{
Norimichi Akiyama ${ }^{1}$, Naoki Inui ${ }^{1,2}$, Kazutaka Mori ${ }^{1}$, Yutaro Nakamura ${ }^{1}$, Hiroshi Hayakawa ${ }^{3}$, \\ Shimako Tanaka ${ }^{4}$, Shinya Uchida ${ }^{4}$, Noriyuki Namiki ${ }^{4}$, Hiroshi Watanabe ${ }^{2}$, Takafumi Suda ${ }^{1}$ \\ ${ }^{1}$ Second Division, Department of Internal Medicine, ${ }^{2}$ Department of Clinical Pharmacology and Therapeutics, Hamamatsu University School \\ of Medicine, Handayama, Hamamatsu, Japan; ${ }^{3}$ Department of Respiratory Medicine, Tenryu Hospital, Oro, Hamamatsu, Japan; ${ }^{4}$ Department of \\ Pharmacy Practice and Science, School of Pharmaceutical Sciences University of Shizuoka, Shizuoka, Japan \\ Contributions: (I) Conception and design: N Akiyama, N Inui; (II) Administrative support: H Watanabe, T Suda; (III) Provision of study materials or \\ patients: N Akiyama, N Namiki, H Watanabe, T Suda; (IV) Collection and assembly of data: N Akiyama, N Inui, S Tanaka, S Uchida, Y Nakamura; (V) \\ Data analysis and interpretation: N Akiyama, N Inui, K Mori; (VI) Manuscript writing: All authors; (VII) Final approval of manuscript: All authors. \\ Correspondence to: Naoki Inui, MD, PhD. Second Division, Department of Internal Medicine, Hamamatsu University School of Medicine, 1-20-1 \\ Handayama, Hamamatsu 431-3192, Japan. Email: inui@hama-med.ac.jp.
}

\begin{abstract}
Background: The prevalence of pulmonary infections caused by nontuberculous mycobacteria (NTM) is increasing worldwide. Furthermore, the treatment of infections caused by the Mycobacterium aviumintracellulare complex (MAC) remains challenging. The cytochrome P450 (CYP) enzyme inducer, rifampicin, and the CYP inhibitor, clarithromycin, have clinical activity against MAC and key drugs in the treatment of MAC infection. The interaction of rifampicin and clarithromycin may influence the therapeutic process.

Methods: Thirty-one Japanese chemo-naïve patients with pulmonary MAC infection were included in the study. Before and after 7-day administration of rifampicin and clarithromycin, the pharmacokinetics of midazolam, a CYP3A-specific probe, were analyzed. The concentrations of midazolam were determined by liquid chromatography-tandem mass spectrometry. None of the patients were receiving any other medications that might affect CYP3A activity.

Results: Of the patients, 24 (77.4\%) were infected with Mycobacterium avium (M. avium) and 7 (22.6\%) were infected with Mycobacterium intracellulare ( $M$. intracellulare). The concentrations of midazolam were significantly reduced with administration of rifampicin and clarithromycin [the median (range) was 1.75 $(0.70-8.22)$ to $1.04(0.30-2.63) \mathrm{ng} / \mathrm{mL}, \mathrm{P}<0.0001]$. The differences in midazolam levels were not correlated with clinical characteristics.
\end{abstract}

Conclusions: Coadministration of rifampicin and clarithromycin may increase CYP3A enzymatic activity.

Keywords: Clarithromycin; cytochrome P450 (CYP); drug interaction; Mycobacterium avium-intracellulare complex (MAC); rifampicin

Submitted Apr 08, 2019. Accepted for publication Aug 30, 2019.

doi: $10.21037 /$ jtd.2019.09.06

View this article at: http://dx.doi.org/10.21037/jtd.2019.09.06

\section{Introduction}

Infections caused by nontuberculous mycobacteria (NTM) are widespread (1-3). In particular, the nodular bronchiectasis type of infection, which typically occurs in the right middle lobe or lingular segment in middle-aged non-immunocompromised hosts who have no underlying pulmonary disease, is increasing in prevalence (1). Although
NTM is ubiquitous in the environment and comprise many species, Mycobacterium avium (M. avium) and Mycobacterium intracellulare (M. intracellulare), usually termed as the Mycobacterium avium-intracellulare complex (MAC), are the most common mycobacteria (1). These two mycobacteria are non-transmissible between humans and have low infectivity $(1,4)$. 
However, the treatment of MAC infection remains challenging. One reason for this is that patients with MAC infection usually have significant comorbidities, underlying or preexisting pulmonary diseases, and immunosuppressive conditions $(1,3)$. In the US Medicare beneficiary survey, patients with pulmonary NTM infection had a mean of four comorbidities (2). Most patients infected with NTM are of the older generation (2). Another challenge in the treatment of MAC infection is that the antibiotics used to treat it have low efficacy against MAC $(1,5)$. Prolonged therapy with multiple antimicrobial agents, including rifampicin, clarithromycin or azithromycin and ethambutol, is routinely recommended for MAC infection. However, the long period of treatment and the high frequency of adverse effects make therapeutic management difficult (5-7).

In addition, drug interactions induced by rifampicin and clarithromycin may also influence the therapeutic process $(1,7)$. Interactions can alter the pharmacokinetics of drugs, induce expected/unexpected adverse reactions, and sometimes abolish clinical efficacy, which is the case for the drug treatment of MAC infection (7). Drug interactions mainly occur through the metabolic pathways of drugs. Cytochrome P450 (CYP) is a major factor involved in drug metabolism $(8,9)$. CYP3A metabolizes a broad range of structurally diverse compounds and consequently plays an important role in many clinically significant drug interactions (10). Rifampicin is a bactericidal antibiotic used for the treatment of both Mycobacterium tuberculosis (M. tuberculosis) and MAC infection $(11,12)$. Rifampicin has potent CYP enzyme-inducing properties (13-16), which can induce clinically significant drug interactions and influence the efficacy of co-administered drugs (17). Clarithromycin has in vitro and clinical activity against MAC and is a key drug in MAC treatment $(12,18)$. In contrast to rifampicin, clarithromycin has CYP3A-inhibiting activity (10), thereby inhibiting the metabolism of co-administered CYP3Adependent drugs and increasing their blood concentration.

In the present study, we aimed to clarify coadministration of rifampicin and clarithromycin-induced CYP3A enzyme activity in patients with pulmonary MAC infection. Usually a single CYP-specific probe is used to assess the CYP phenotype and we used midazolam as a probe drug for CYP3A in this study. Midazolam is exclusively metabolized by CYP3A, which means that midazolam is a suitable and commonly used in vivo probe for CYP3A activity. Previous pharmacokinetic studies reported that midazolam levels at $4 \mathrm{~h}$ were related to CYP3A activity $(19,20)$. In addition, we examined the concentrations of rifampicin and clarithromycin.

\section{Methods}

\section{Subjects}

This study was conducted in accordance with the Declaration of Helsinki and was approved by the Institutional Review Board of each participating institution (Hamamatsu, Japan, approval number: 17-022). Each patient was informed of and understood the purpose, methods and risks of this research. Each patient provided written informed consent to be included in the study. The study was registered with the University Hospital Medical Information Network Clinical Trial Registry (UMIN ID 000027308).

The inclusion criteria for the study were as follows: patients who were aged 20 years or older, with microbiologic and radiographic evidence of MAC infection. Patients were diagnosed with pulmonary MAC disease based on the Japanese Society for Tuberculosis statement (21), and had no prior history of treatment for any mycobacterial infection. Key exclusion criteria included severe hepatic and renal dysfunction and a history of allergy or hypersensitivity to the study drugs. Patients who had taken any supplements, herbs, St John's wort, grapefruit, or any drugs likely to interact with the study medications within the previous week were excluded.

\section{Study design}

Rifampicin was administered to patients $(10 \mathrm{mg} / \mathrm{kg}$, to a maximum dose of $450 \mathrm{mg}$; Rifadin capsules; Daiichi Sankyo Co., Ltd., Tokyo, Japan) once daily in the morning, as was clarithromycin $(20 \mathrm{mg} / \mathrm{kg}$, to a maximum dose of $800 \mathrm{mg}$; Clarith; Taisho Toyama Pharmaceutical Co., Ltd., Tokyo, Japan) These are the usual dosages administered in Japan. Before and after 7-day administration of rifampicin and clarithromycin, a pharmacokinetics study of midazolam was performed. Blood samples were obtained before and $4 \mathrm{~h}$ after the administration of midazolam $(15 \mu \mathrm{g} / \mathrm{kg}$; Dormicum; Astellas Pharma Inc., Tokyo, Japan), and centrifuged at 3,000 rpm for $10 \mathrm{~min}$ at $4{ }^{\circ} \mathrm{C}$. The blood samples were stored at $-80^{\circ} \mathrm{C}$ until analysis. Before and after each trial, blood samples were also taken for standardized safety assessments. Clinical, radiological and laboratory data at the time of diagnosis were obtained from medical records. After this study, patients received ethambutol and/ or streptomycin. Other drugs that have no CYP3A inducing 
or inhibiting activity were used at the same dose and dosage schedule throughout the study period.

\section{Measurement of midazolam}

Measurements of midazolam were performed by liquid chromatography-tandem mass spectrometry, as described previously $(16,20)$. Briefly, a $5-\mu \mathrm{L}$ aliquot of each sample was analyzed in a TSQ7000C (Thermo Electron, Waltham, MA, USA) equipped with a spectra system (Thermo Electron, Waltham, MA, USA). Chromatographic separation was achieved under isocratic conditions using a C18 column with a guard column (Symmetry; $2.1 \times 10 \mathrm{~mm}$; $5 \mu \mathrm{L}$; Waters, Milford, MA, USA). The mobile phase for the assay consisted of methanol and $10 \mathrm{mM}$ ammonium acetate $(60: 40, v / v)$ delivered at $0.2 \mathrm{~mL} / \mathrm{min}$. The mass spectrometer was operated in electrospray ionization mode with positive-ion detection mode. Ion transitions were monitored from mass-to-charge ratio $(\mathrm{m} / \mathrm{z}) 326.0$ to $\mathrm{m} / \mathrm{z}$ 291.0 for midazolam and $\mathrm{m} / \mathrm{z} 282.0$ to $\mathrm{m} / \mathrm{z} 236.0$ for nitrazepam (internal standard). These transitions represent the product ions of $[\mathrm{M}-\mathrm{H}]^{+}$ions. The limit of quantification was $0.1 \mathrm{ng} / \mathrm{mL}$, and the method was linear from 0.1 to $100 \mathrm{ng} / \mathrm{mL}$. The intra-assay coefficient of variation was $<8.9 \%$. Data acquisition and analysis were performed using the Analyst software (version 1.4.1; AB Sciex, Framingham, MA, USA). Midazolam plasma concentrations were used to assess CYP3A activity $(19,20)$ and changes in rifampicin and clarithromycin-induced CYP3A activity were assessed by comparing the concentration of midazolam in plasma samples $(16,19)$.

\section{Measurement of the plasma concentrations for each drug}

Samples were collected before, and 2 and $6 \mathrm{~h}$ after, administration of rifampicin and clarithromycin. The concentrations of rifampicin and clarithromycin were measured using liquid chromatography-tandem mass spectrometry with an Alliance 2695 system (Waters Co., MA, USA) coupled with a CAPCELL PAK C18AQ column $(150 \times 2 \mathrm{~mm}$; id, $5 \mu \mathrm{m}$; Shiseido, Tokyo, Japan) and a Waters Sentry guard column $(10 \times 2.1 \mathrm{~mm}$; id, $0.35 \mu \mathrm{m}$; Waters Co., MA, USA). The mobile phase used ammonium acetate (5 mM): acetonitrile (50:50) with elution at a flow rate of $0.2 \mathrm{~mL} / \mathrm{min}$ at $40^{\circ} \mathrm{C}$ for $10 \mathrm{~min}$. The mass spectrometry analysis was performed in the positive ion mode used a Micromass ZQ (Waters Co., MA, USA) at the following transitions: $748.5 \mathrm{~m} / \mathrm{z}$ for clarithromycin and $823.4 \mathrm{~m} / \mathrm{z}$ for rifampicin. The pharmacokinetic parameters were estimated by compartmental analysis from the concentration-time profile in plasma. The area under the plasma concentrationtime curve from 0 to $6 \mathrm{~h}\left(\mathrm{AUC}_{0-6}\right)$ was calculated by the trapezoidal rule.

\section{Statistical analysis}

Correlations between continuous variables were undertaken using Spearman's rank correlation coefficient. Partial correlation analyses were performed with adjustments for age. Data are expressed as the median and range unless indicated otherwise. A $\mathrm{P}$ value $<0.05$ was considered significant. All statistical analyses were performed with EZR (Saitama Medical Center, Jichi Medical University, Saitama, Japan), which is a graphical user interface for R (The R Foundation for Statistical Computing, Vienna, Austria).

\section{Results}

\section{Patient characteristics}

From July 2017 to May 2018, 31 Japanese patients, who were diagnosed with pulmonary MAC and received rifampicin and clarithromycin, were enrolled in this study (Table 1). The median age at diagnosis was 68 years (range, 48-84 years), with a duration of 1.8 months (range, 0-7 months) from diagnosis to enrollment. Twenty-four patients $(77.4 \%)$ were women. The median body mass index was 18.8 (range, 14.2-26.5). Seventeen patients (54.8\%) showed symptoms: 14 had a cough, 10 had sputum, 2 had fatigue, and 2 had dyspnea. The others showed no symptoms but abnormal findings were detected by chest $\mathrm{X}$-ray examination. Some patients had preexisting structural pulmonary diseases, one had chronic obstructive pulmonary disease (COPD), one had pleural thickening, and 24 had bronchiectasis for which a link with MAC infection could not be ruled out. Four patients had a malignancy and 6 had diabetes mellitus. None were positive for human immunodeficiency virus (HIV) infection. Twenty-four (77.4\%) patients were infected with M. avium and 7 (22.6\%) were infected with $M$. intracellulare. None of the patients used other medications that affected CYP3A activity. The median dose of clarithromycin was $800 \mathrm{mg}$.

\section{Pharmacokinetic studies}

There were no significant correlations detected between 
Table 1 Patient characteristics

\begin{tabular}{|c|c|}
\hline Characteristics & All patients $(n=31)$ \\
\hline Age, years [range] & 68 [48-84] \\
\hline Sex, male/female & $7 / 24$ \\
\hline Height [cm] & $155[137-174]$ \\
\hline Weight (kg) & $46.0(32.5-73.5)$ \\
\hline Body mass index $\left(\mathrm{kg} / \mathrm{m}^{2}\right)$ & $18.8(14.2-26.5)$ \\
\hline Non-smoker & $26(83.9)$ \\
\hline $\begin{array}{l}\text { Duration from diagnosis to treatment } \\
\text { [months] }\end{array}$ & $1.8[0-7]$ \\
\hline \multicolumn{2}{|l|}{ Etiology } \\
\hline Mycobacterium avium & $24(77.4)$ \\
\hline Mycobacterium intracellulare & 7 (22.6) \\
\hline \multicolumn{2}{|l|}{ Dose of treatment [mg] } \\
\hline Clarithromycin & $800[600-800]$ \\
\hline Rifampicin & $450[300-450]$ \\
\hline \multicolumn{2}{|l|}{ CT findings } \\
\hline Nodular bronchiectasis type & 24 \\
\hline Solitary nodular shadow & 3 \\
\hline Cavity & 4 \\
\hline \multicolumn{2}{|l|}{ Laboratory findings } \\
\hline White blood cells & $5,100[3,440-12,290]$ \\
\hline C-reactive protein & $0.07(0.02-8.32)$ \\
\hline
\end{tabular}

Data are expressed as the number (\%) or median (range) unless otherwise indicated. CT, computed tomography.

midazolam levels and sex (Table 2, $\mathrm{P}=0.31$ ), body mass index $(\mathrm{r}=-0.12, \mathrm{P}=0.52)$, or the presence of a comorbidity $(\mathrm{P}=0.57)$, but a correlation was detected with age $(\mathrm{r}=0.49, \mathrm{P}=0.006)$.

When the concentrations of midazolam were compared before and after 7-day of administration of rifampicin and clarithromycin, a significant reduction was detected from $1.75(0.70-8.22)$ to $1.04(0.30-2.63)$ (Figure $1, \mathrm{P}<0.001)$. The levels of midazolam were decreased in 28 patients and elevated in three patients. We assessed the correlations between the changes in midazolam level and clinical parameters (Table 3). To exclude interdependence between age and midazolam levels, we performed partial correlation analyses, which demonstrated that age and the differences in midazolam concentration were not correlated.

Plasma samples for rifampicin and clarithromycin pharmacokinetic analysis were obtained after 7-day
Table 2 Correlations between the plasma midazolam levels and clinical parameters

\begin{tabular}{lcc}
\hline Characteristics & $r$ & P value \\
\hline Age & 0.49 & 0.006 \\
Sex, female & 0.19 & 0.31 \\
Height (cm) & -0.22 & 0.24 \\
Weight (kg) & -0.14 & 0.46 \\
Smoker & -0.34 & 0.06 \\
Presence of Mycobacterium avium & 0.16 & 0.40 \\
Presence of comorbidity & 0.12 & 0.53 \\
Asthma & -0.18 & 0.32 \\
COPD & 0.27 & 0.15 \\
Malignancy & -0.12 & 0.53 \\
Diabetes mellitus & 0.29 & 0.11 \\
Presence of a cavity on X-ray & 0.10 & 0.60 \\
\hline
\end{tabular}

Data are expressed as the Spearman's rank correlation coefficient $(r)$ and $\mathrm{P}$ value. COPD, chronic obstructive pulmonary disease.

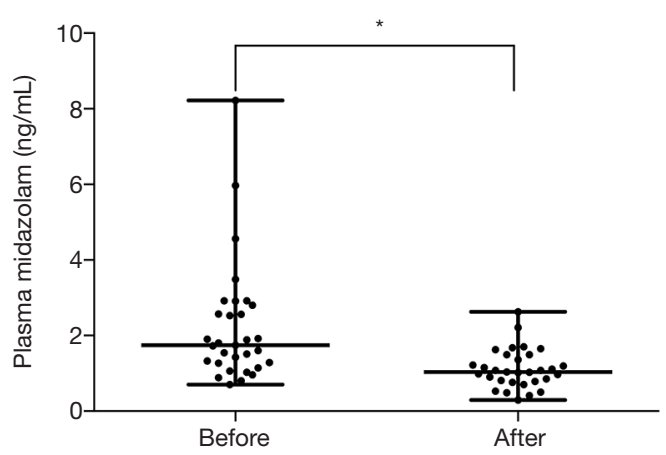

Figure 1 Concentrations of midazolam before and after 7-day administration of rifampicin and clarithromycin. The midazolam levels were significantly reduced after administration of rifampicin and clarithromycin $\left.{ }^{*}, \mathrm{P}<0.001\right)$. Horizontal lines represent the median, maximum and minimum values.

administration of both drugs. The concentrations of rifampicin and clarithromycin at $2 \mathrm{~h}$ were $14.10(3.75-$ $21.75) \mathrm{ng} / \mathrm{mL}$ and $0.77(0.19-3.67) \mathrm{ng} / \mathrm{mL}$, respectively. The $\mathrm{AUC}_{0-6}$ values for rifampicin and clarithromycin were 55.06 (22.69-95.94) and 2.72 (0.81-12.91). Although clinical characteristics, such as age, sex and bacterial species did not relate to the pharmacokinetic parameters of clarithromycin, the concentration of clarithromycin at $2 \mathrm{~h}$ 
Table 3 Partial correlations between the differences in plasma midazolam levels and clinical parameters

\begin{tabular}{lcc}
\hline Characteristics & $\mathrm{r}$ & $\mathrm{P}$ value \\
\hline Sex, female & -0.23 & 0.22 \\
Height $(\mathrm{cm})$ & -0.06 & 0.76 \\
Weight $(\mathrm{kg})$ & 0.31 & 0.09 \\
Smoker & 0.02 & 0.91 \\
Presence of Mycobacterium avium & -0.22 & 0.23 \\
Presence of comorbidity & & \\
Asthma & -0.09 & 0.62 \\
COPD & -0.32 & 0.08 \\
Malignancy & -0.12 & 0.53 \\
Diabetes mellitus & 0.17 & 0.36 \\
Presence of a cavity on X-ray & -0.32 & 0.99 \\
\hline
\end{tabular}

Data are expressed as the correlation coefficient $(r)$ and $P$ value. Partial rank correlations were adjusted with age. COPD, chronic obstructive pulmonary disease.

significantly correlated with the midazolam level after 7-day administration (Figure 2, $\mathrm{r}=0.452, \mathrm{P}=0.011$ ).

\section{Discussion}

In the present study, we investigated the changes in CYP3A enzymatic activity following the administration of rifampicin and clarithromycin, CYP3A inducer and CYP3A inhibitor, respectively, in patients with pulmonary MAC infection. In pharmacokinetic studies using midazolam as a probe drug, the concentrations of midazolam were decreased, which indicated that CYP3A enzymatic activity was increased. The changes in CYP3A following the administration of rifampicin and clarithromycin might affect the efficacy and safety of medications against MAC infection.

Drug interactions can result in altered drug concentrations and insufficient antibiotic exposure. Some previous reports have shown pharmacokinetic changes in rifampicin and clarithromycin during combination therapy against MAC infection. In a large cohort of patients with pulmonary MAC infection, concurrent administration of rifampicin led to a $68 \%$ decrease in the peak concentration of clarithromycin (7). Yamamoto et al. administered low doses of clarithromycin (400-600 mg per a day) to nine MAC infection patients and measured the plasma concentrations of clarithromycin and its metabolite 14-R-hydroxyclarithromycin (22). They
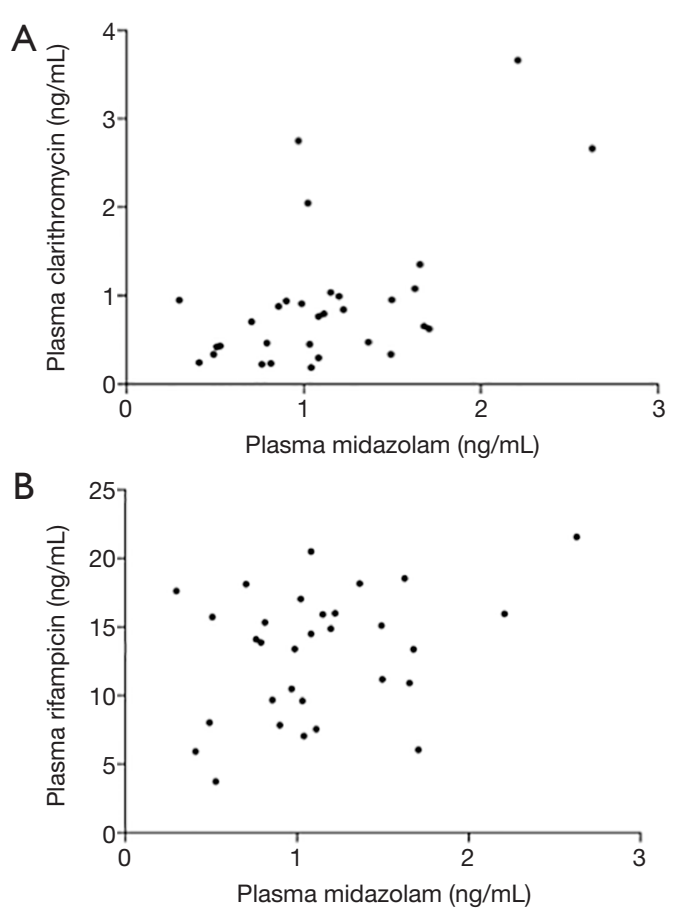

Figure 2 Correlations between the concentrations of midazolam and clarithromycin (A) and rifampicin (B) after 7 -day administration of rifampicin and clarithromycin. The concentration of clarithromycin at $2 \mathrm{~h}$ significantly correlated with the midazolam level after 7-day administration ( $\mathrm{r}=0.452, \mathrm{P}=0.011)$. The concentration of rifampicin at $2 \mathrm{~h}$ was not correlated with the midazolam level $(\mathrm{r}=0.240, \mathrm{P}=0.201)$.

found that the mean concentration of clarithromycin was significantly decreased ( one fifth) in the presence of rifampicin. Koh et al. performed a pharmacokinetic study of clarithromycin and showed that plasma clarithromycin concentrations were significantly lower in patients who received both clarithromycin and rifampicin compared with those who received clarithromycin without rifampicin (23). Whereas rifampicin reportedly lowered clarithromycin levels, the effect of clarithromycin on the pharmacokinetics of rifampicin was unclear. van Ingen et al. investigated the effect of clarithromycin on rifampicin and the concentration of rifampicin remained unaffected by coadministration of macrolides (7). By contrast, when clarithromycin was added to rifampicin instead of streptomycin in patients infected with another NTM, Mycobacterium ulcerans, a $60 \%$ increase in the AUC for rifampicin was reported (24).

Drug interactions between rifampicin and clarithromycin are related to CYP3A enzymatic activity, but investigations targeting CYP3A enzymatic activity during the 
coadministration of rifampicin and clarithromycin were lacking. To clarify the interaction between rifampicin and clarithromycin, we carried out an investigation using midazolam as a probe drug for CYP3A. In the present study, we found that coadministration of rifampicin and clarithromycin reduced the levels of midazolam, indicating that this drug combination increased CYP3A activity. Shimomura et al. examined the urinary concentrations of endogenous cortisol and $6 \beta$-hydroxycortisol in nine pulmonary MAC patients administered clarithromycin and rifampicin (25). The mean $6 \beta$-hydroxycortisol/ cortisol ratio, a CYP3A4 activity marker, increased after administration of rifampicin and clarithromycin in this study, albeit with a small sample size. Although the CYP3A inducer, rifampicin, and the CYP3A inhibitor, clarithromycin, induced bidirectional changes in CYP3A activity, the results presented above and those of our study suggested that the effects of rifampicin were more marked than those of clarithromycin.

In the present study, we examined the levels of clarithromycin $2 \mathrm{~h}$ after clarithromycin administration because this is reported to be the peak concentration time for this drug following administration (22). The median concentration of clarithromycin at $2 \mathrm{~h}$ was $0.78 \mu \mathrm{g} / \mathrm{mL}$, which correlated with the midazolam level after 7-day administration. The clarithromycin minimum inhibitory concentration (MIC) for MAC infection was in the range of $1-4 \mu \mathrm{g} / \mathrm{mL}$, which was achievable by administration of $800-1,000 \mathrm{mg}$ of the drug (1). The concentrations of clarithromycin were high in phagocytes and lung tissue compared with serum. Although both the optimal dose of clarithromycin to treat MAC infection (12) and the clinical significance of the marked reduction in the serum level of clarithromycin in the treatment of MAC remain unclear, a suboptimal plasma concentration of clarithromycin might be one of the reasons for an inadequate response to macrolide-based therapy.

To avoid, or at least limit, drug interactions, two approaches have been tried in the treatment of MAC infection. One is the use of rifabutin (1). Rifabutin is another rifampin that has stronger activity against mycobacteria than rifampicin itself (26). Some guidelines recommend the use of rifabutin in the treatment of NTM infections $(1,27)$. However, rifabutin is also an inducer of CYP3A and the interaction between rifabutin and clarithromycin must be taken into consideration, although its magnitude of induction is less than that of rifampicin. Hafner et al. performed a pharmacokinetic study of rifabutin and clarithromycin in HIV-positive patients (28). Clarithromycin increased the rifabutin concentration and this was accompanied by the increased adverse effects of rifabutin.

The other approach is to treat without rifampicin. We conducted a pilot study to compare rifampicin-containing triple therapy with clarithromycin and ethambutol double therapy without rifampicin in patients with pulmonary MAC disease (29). Patients with high-resolution computed tomography (CT)-detected cavitation were less than half of the patient sample. We set the primary endpoint as the conversion of sputum cultures from MAC positive to negative after 12 months of treatment. The rate of sputum culture conversion was $40.6 \%$ with the rifampicincontaining regimen compared with $55.0 \%$ with the no rifampicin regimen, which confirmed the effectiveness of the regimen lacking rifampicin. The incidence of adverse events leading to the discontinuation of treatment was $37.2 \%$ in the rifampicin-containing regimen compared with $26.6 \%$ in the no rifampicin regimen. We concluded that treatment with clarithromycin and ethambutol showed comparative efficacy in terms of the conversion of sputum cultures to negative and avoided the discontinuation of medication because of adverse events, although the results need to be verified in large and various regional populations.

There are some limitations in the present study. First, we did not examine the effect of p-glycoprotein. Rifampicin induces the p-glycoprotein efflux transporter and is the substrate of p-glycoprotein, clarithromycin inhibits $\mathrm{p}$-glycoprotein. The drug interactions between rifampicin and clarithromycin may be related to p-glycoproteinmediated activity. Second, CYP3A activity was not increased in all patients. The effects of rifampicin and clarithromycin may depend on patient characteristics and endogenous CYP activity. In the present study, a significant correlation was detected between midazolam levels and age. Using partial rank correlation analysis, we demonstrated that administration of rifampicin and clarithromycin reduced midazolam levels independently of age. Third, we did not assess drug interactions between rifampicin and another macrolide, azithromycin, which has a lower affinity for CYP (30). There is no reported difference between clarithromycin and azithromycin in the management of pulmonary MAC infection (1) and the inclusion of azithromycin may be appropriate for MAC therapy involving rifampicin. However, clinicians should pay attention to drug interactions with rifampicin because this drug combination will increase CYP3A activity. van Ingel $e t$ 
al. showed that coadministration of rifampicin reduced the maximum plasma concentration of azithromycin by $23 \%$ (7).

Coadministration of rifampicin and clarithromycin increased CYP3A enzymatic activity. This might partly explain the poor outcomes of currently recommended treatment regimens. Drug interactions between rifampicin and clarithromycin may cause significant changes in the systemic exposure of both compounds and their metabolites, and this may have important implications for the safety and effectiveness of therapy against MAC.

\section{Acknowledgments}

We thank Kate Fox, DPhil, from Edanz Group (www. edanzediting.com/ac) for editing a draft of this manuscript.

\section{Footnote}

Conflicts of Interest: The authors have no conflicts of interest to declare.

Ethical Statement: The authors are accountable for all aspects of the work in ensuring that questions related to the accuracy or integrity of any part of the work are appropriately investigated and resolved. This study was conducted in accordance with the Declaration of Helsinki and was approved by the Institutional Review Board of each participating institution (Hamamatsu, Japan, approval number: 17-022). Each patient was informed of and understood the purpose, methods and risks of this research. Each patient provided written informed consent to be included in the study. The study was registered with the University Hospital Medical Information Network Clinical Trial Registry (UMIN ID 000027308).

\section{References}

1. Griffith DE, Aksamit T, Brown-Elliott BA, et al. An official ATS/IDSA statement: diagnosis, treatment, and prevention of nontuberculous mycobacterial diseases. Am J Respir Crit Care Med 2007;175:367-416.

2. Adjemian J, Olivier KN, Seitz AE, et al. Prevalence of nontuberculous mycobacterial lung disease in U.S. Medicare beneficiaries. Am J Respir Crit Care Med 2012;185:881-6.

3. Satta G, McHugh TD, Mountford J, et al. Managing pulmonary nontuberculous mycobacterial infection. time for a patient-centered approach. Ann Am Thorac Soc
2014;11:117-21.

4. Horsburgh CR Jr. Mycobacterium avium complex infection in the acquired immunodeficiency syndrome. $\mathrm{N}$ Engl J Med 1991;324:1332-8.

5. Tanaka E, Kimoto T, Tsuyuguchi K, et al. Effect of clarithromycin regimen for Mycobacterium avium complex pulmonary disease. Am J Respir Crit Care Med 1999;160:866-72.

6. Huang JH, Kao PN, Adi V, et al. Mycobacterium aviumintracellulare pulmonary infection in HIV-negative patients without preexisting lung disease: diagnostic and management limitations. Chest 1999;115:1033-40.

7. van Ingen J, Egelund EF, Levin A, et al. The pharmacokinetics and pharmacodynamics of pulmonary Mycobacterium avium complex disease treatment. Am J Respir Crit Care Med 2012;186:559-65.

8. Zhang QY, Dunbar D, Ostrowska A, et al. Characterization of human small intestinal cytochromes P-450. Drug Metab Dispos 1999;27:804-9.

9. Guengerich FP. Cytochrome P-450 3A4: regulation and role in drug metabolism. Annu Rev Pharmacol Toxicol 1999;39:1-17.

10. Quinney SK, Haehner BD, Rhoades MB, et al. Interaction between midazolam and clarithromycin in the elderly. $\mathrm{Br} \mathrm{J}$ Clin Pharmacol 2008;65:98-109.

11. Blumberg HM, Burman WJ, Chaisson RE, et al. American thoracic society/centers for disease control and prevention/ infectious diseases society of America: treatment of tuberculosis. Am J Respir Crit Care Med 2003;167:603-62.

12. Shafran SD, Singer J, Zarowny DP, et al. A comparison of two regimens for the treatment of Mycobacterium avium complex bacteremia in AIDS: rifabutin, ethambutol, and clarithromycin versus rifampin, ethambutol, clofazimine, and ciprofloxacin. Canadian HIV trials network protocol 010 study group. N Engl J Med 1996;335:377-83.

13. Backman JT, Olkkola KT, Neuvonen PJ. Rifampin drastically reduces plasma concentrations and effects of oral midazolam. Clin Pharmacol Ther 1996;59:7-13.

14. Gorski JC, Vannaprasaht S, Hamman MA, et al. The effect of age, sex, and rifampin administration on intestinal and hepatic cytochrome P450 3A activity. Clin Pharmacol Ther 2003; 74:275-87.

15. Backman JT, Kivistö KT, Olkkola KT, et al. The area under the plasma concentration-time curve for oral midazolam is 400 -fold larger during treatment with itraconazole than with rifampicin. Eur J Clin Pharmacol 1998;54:53-8.

16. Inui $\mathrm{N}$, Akamatsu $\mathrm{T}$, Uchida $\mathrm{S}$, et al. Chronological effects 
of rifampicin discontinuation on cytochrome $\mathrm{P} 450$ activity in healthy Japanese volunteers, using the cocktail method. Clin Pharmacol Ther 2013;94:702-8.

17. Niemi M, Backman JT, Fromm MF, et al. Pharmacokinetic interactions with rifampicin: clinical relevance. Clin Pharmacokinet 2003;42:819-50.

18. Wallace RJ Jr, Brown BA, Griffith DE, et al. Clarithromycin regimens for pulmonary Mycobacterium avium complex. The first 50 patients. Am J Respir Crit Care Med 1996;153:1766-72.

19. Ryu JY, Song IS, Sunwoo YE, et al. Development of the "Inje cocktail" for high-throughput evaluation of five human cytochrome $\mathrm{P} 450$ isoforms in vivo. Clin Pharmacol Ther 2007;82:531-40.

20. Misaka S, Uchida S, Imai H, et al. Pharmacokinetics and pharmacodynamics of low doses of midazolam administered intravenously and orally to healthy volunteers. Clin Exp Pharmacol Physiol 2010;37:290-5.

21. Nontuberculous Mycobacteriosis Control Committee of the Japanese Society for Tuberculosis., Scientific Assembly for Infection and Tuberculosis of the Japanese Respiratory Society., International Exchanging Committee of the Japanese Society for Tuberculosis. Guidelines for the diagnosis of pulmonary nontuberculous mycobacterial diseases--2008. Kekkaku 2011;86:37-9.

22. Yamamoto F, Harada S, Mitsuyama T, et al. Concentration of clarithromycin and 14-R-hydroxy-clarithromycin in plasma of patients with Mycobacterium avium complex infection, before and after the addition of rifampicin. Jpn J Antibiot 2004;57:124-33.

23. Koh WJ, Jeong BH, Jeon K, et al. Therapeutic drug monitoring in the treatment of Mycobacterium avium

Cite this article as: Akiyama N, Inui N, Mori K, Nakamura Y, Hayakawa H, Tanaka S, Uchida S, Namiki N, Watanabe H, Suda T. Effect of rifampicin and clarithromycin on the CYP3A activity in patients with Mycobacterium avium complex. J Thorac Dis 2019;11(9):3814-3821. doi: 10.21037/jtd.2019.09.06 complex lung disease. Am J Respir Crit Care Med 2012;186:797-802.

24. Alffenaar JW, Nienhuis WA, de Velde F, et al. Pharmacokinetics of rifampin and clarithromycin in patients treated for Mycobacterium ulcerans infection. Antimicrob Agents Chemother 2010;54:3878-83.

25. Shimomura H, Andachi S, Aono T, et al. Serum concentrations of clarithromycin and rifampicin in pulmonary Mycobacterium avium complex disease: long-term changes due to drug interactions and their association with clinical outcomes. J Pharm Health Care Sci 2015;1:32.

26. Saito H, Sato K, Tomioka H. Comparative in vitro and in vivo activity of rifabutin and rifampicin against Mycobacterium avium complex. Tubercle 1988;69:187-92.

27. Haworth CS, Banks J, Capstick T, et al. British thoracic society guidelines for the management of non-tuberculous mycobacterial pulmonary disease (NTM-PD). Thorax 2017;72:ii1-64.

28. Hafner R, Bethel J, Power M, et al. Tolerance and pharmacokinetic interactions of rifabutin and clarithromycin in human immunodeficiency virus-infected volunteers. Antimicrob Agents Chemother 1998;42:631-9.

29. Miwa S, Shirai M, Toyoshima M, et al. Efficacy of clarithromycin and ethambutol for Mycobacterium avium complex pulmonary disease. A preliminary study. Ann Am Thorac Soc 2014;11:23-9.

30. Westphal JF. Macrolide-induced clinically relevant drug interactions with cytochrome P-450A (CYP) 3A4: an update focused on clarithromycin, azithromycin and dirithromycin. Br J Clin Pharmacol 2000;50:285-95. 